\title{
Serial learning of nonsense shapes as a function of instructions controlling labeling*
}

\author{
LINDA S. JAMES \\ Indiana University, Bloomington, Indiana 47401
}

\begin{abstract}
High- or low-complexity nonsense shapes were used in a serial learning task involving a recognition response. Ss were instructed either to provide their own labels as a learning aid or to learn the shapes (with no mention of labeis), or they were inhibited from labeling by a simultaneous shadowing task. Shadowing retarded learning of the serial task, but there were no reliable differences among the other four groups. No reliable differences among groups were observed on a subsequent mediation task. Within each group, those shapes which Ss denied labeling had no more errors than those which Ss reported labeling. The effectiveness of shadowing as a means of inhibiting labeling was attested to by the large number of Ss in these groups who denied the use of labels.
\end{abstract}

Examination of the stimulus predifferentiation literature reveals an extremely complex relationship governing the relative facilitative effects of labeling and observation pretraining on transfer to a recognition or discrimination task. Epstein (1967) and Ellis (1969) have discussed the difficulties involved in interpreting these data. In a typical predifferentiation study (cf. Ellis \& Muller, 1964), Ss given labeling pretraining received a fixed number of paired-associate trials (with feedback) in which to learn a label response (E-supplied) to a shape stimulus. Observation pretraining consists of presenting the shapes for the same number of trials under instructions to inspect and discriminate among them; no feedback is given. Following this pretraining, Ss are transferred to a recognition task requiring a discrimination between an "old" shape and highly similar "new" shapes. This predifferentiation procedure, as Malloy \& Ellis (1970) note, does not insure comparable levels of attention during pretraining; nor does the procedure provide comparable feedback about the correctness of discrimination during pretraining. Consequently, significant recognition transfer effects due to labeling pretraining are not necessarily due to the effects of a label, per se.

On the other hand, the lack of a pretraining effect does not necessarily mean that labels do not facilitate recognition. Some Ss will assign their own labels to the shapes during observation pretraining; if implicit labeling is sufficiently widespread, then the comparison becomes one between E-supplied and S-supplied labeling groups.

Then, too, as Epstein (1967) stresses, it is important to consider the similarity of the contexts of paired-associate pretraining and transfer tasks of

\footnotetext{
*Based upon a dissertation submitted in partial fulfillment of the requirements for the $\mathrm{PhD}$ degree at Indiana University. This research was supported in part by NSF Grant GB 3977 to Indiana University Foundation. L. R. Peterson, principal investigator. I wish to thank L. R. Peterson and C. T. James for their suggestions.
}

discrimination. The two are quite different, and Epstein argues that it is legitimate to wonder to what extent the labels learned in one context will transfer to the other.

We see, then, that there are three difficulties incurred by the typical predifferentiation study: different task demands during pretraining can lead to differences in attention; implicit labeling is not controlled (and customarily no attempt is made to assess the extent to which it occurs); finally, there is a differing amount of nonspecific transfer between the transfer task and the various pretraining tasks.

The current paper presents an alternative methodology which circumvents some of these problems. Ss were required to learn a serial list of nonsense shapes and were given instructions differing in the extent to which they were expected to elicit labeling. Labeling was encouraged in one set of Ss (Group L) through explicit instructions to label. Other Ss (Group N) received neutral instructions containing no mention of learning strategies. Implicit labeling was inhibited in a third group of Ss (Group Sh) by giving them prose passages to shadow during learning. The task requirements were the same for all Ss-the learning of a serial anticipation task to a common criterion of performance. It was expected that implicit labeling would be most pronounced in Group L, intermediate in Group N, and minimal for Group Sh.

Several problems are averted through the use of this methodology. First, no pretraining was given, so there were no problems of interpretation concerning similarity of the pretraining and transfer tasks. Those Ss who label and those who do not remain in the same experimental context throughout. Secondly, all Ss, whether they label or not, should attend to all shapes equally and receive the same amount of feedback; the task requirements are the same for all Ss. Thirdly, some control over" implicit labeling was gained through instructions. To determine just how much, two other pieces of data were collected after the list was learned. An association task was given in which we measured time to give an association to each 
stimulus shape. It was assumed that association latencies would be shorter for those Ss who labeled. Following the assaciation task, Ss were asked whether they had used labels while learning the serial list.

We think this paradigm is a valid one for examining the role of labels upon recognition learning of nonsense shapes, but realize that there are differences between this serial recognition task and the classical predifferentiation task. Given that labeling has been shown to facilitate recognition of shapes in the latter paradigm, the present study is interested in whether labeling will facilitate serial-position recognition learning.

There is good reason to suppose labels will not facilitate performance in the serial-position task. Daniel and Ellis (1972) and Hake and Erikson (1955) have argued that the advantage of label pretraining (in the predifferentiation paradigm) is to direct S's attention towards those aspects most helpful in discriminating that shape from the others. Since the serial recognition task requires that attention be paid to all shapes, regardless of whether labeled or not, Ss would be expected to identify the crucial aspects for discrimination. Hence, it is likely that labels will not facilitate performance.

Since a comparison of recognition accuracy for rabeled and nonlabeled shapes depends upon their complexity, lists of low- and high-complexity shapes were used under each set of instructions. The primary prediction involved a comparison of the effects of labeling and neutral instructions on the learning of the simple and complex shapes. If groups given neutral instructions contain fewer labeling Ss and if labels facilitate recognition of complex but not of simple shapes, Group L should learn the high-complexity list with fewer errors than Group N, but Group N should commit fewer errors on the low-complexity list. These predictions follow from earlier results (e.g., Ellis \& Muller, 1964). However, if we are correct in claiming that labels are superfluous in this task, then the interaction should not be significant.

\section{METHOD}

\section{Materials}

The stimuli were five 24-point shapes and five 6-point shapes from Vanderplas and Garvin (1959), selected for similar total area; none had an association value greater than $38 \%$. The identification numbers used by Vanderplas and Garvin for the 6-point shapes were $19,20,21,23$, and 25; for the 24-point shapes, $24,25,28,29$, and 30 .

The shapes were photographed with high-contrast copy film, and the negatives were projected as $35-\mathrm{mm}$ slides, giving a white figure on a black background. Two types of slides were constructed: one with a single shape in the center (study slides) and the other with one shape in the center and a different one in each of the four corners (test slides). A Kodak Carousel slide projector was used to project the shapes onto the back of an opaque glass screen placed on a table in front of $S$. At each corner of the screen on S's side was a small lighted button for responding.

\section{Procedure}

A trial was defined as a study series followed by a test series. Study slides were presented at a 2 -sec rate, followed by a test series which was $S$-paced. For example, the study series A, B, C, $D, E, A$ would be followed by the test series beginning with $B$ as the center shape and continuing with Center Shapes C, D, E, A. (On the test sequence, $S$ was instructed to select the shape which followed the center shape by pressing the button in the corner beside that shape.) This test series was followed by the study series B, C, D, E, A, B, and so on, never breaking the serial chain until $S$ met criterion of three consecutive errorless test series. Thus, $\mathrm{S}$ received feedback throughout every test series, as the center shape on one slide was the correct response for the preceding slide. The list was presented in this particular fashion in hopes of eliminating serial position effects.

Five different shape orders were used for each group. On the test slides, the correct shape was placed in each corner once in every four trials, and a particular button was not consistently paired with any stimulus.

An example of the procedure was given using simple shapes (e.g., square, triangle) drawn on index cards. Following the example, Ss in Group L were told that the shapes they had just seen were very simple ones and that therefore one could easily remember their order. They were told further that the shapes they must learn would be more complex, and to enable them to learn the order of the shapes more easily, it would be helpful to associate a word or words with each shape: Group $\mathbf{N}$ and Group Sh were shown the same example to clarify the procedure, but no mention of associations was made, nor were the simple shapes referred to by labels such as "square" or "circle."

\section{Shadowing}

Group Sh was first given instructions for shadowing and received practice in shadowing a paragraph; Ss were told that it was important to shadow as accurately as possible with no pausing. Paragraphs of varying lengths on assorted topics were recorded on tape and presented over earphones. The material was recorded at the rate of approximately 4 syllables/sec with no pausing between paragraphs. The volume was sufficiently loud to allow $S$ to hear the material over his own repetition of it. Shadowing was monitored over an intercom; if $S$ paused in shadowing, he was warned, by the illumination of a small red light located directly above the screen, to continue. Ss in Group Sh were then given the same instructions as those in Group N

After $S$ had been given instructions, the room lights were turned off and $E$ went into the adjoining room to start the slides (and tape recorder for Group Sh). Ss in Group Sh began shadowing before the first study slide was presented and continued until they reached criterion on the serial task. In addition, it was deemed necessary to give these $S$ s a $1-m i n$ rest after 13 trials or $150 \mathrm{ft}$ of tape, whichever occurred first (usually 13 trials), unless $S$ made no errors on the 13 th trial. In that case, $S$ was given a rest following the first error trial. During the rest period, the recorder and projector were turned off, leaving $S$ in darkness except for the four illuminated response buttons.

\section{Mediation}

After $\mathrm{S}$ had reached criterion, he was given a task in which he was required to choose the second shape following the center shape on the test slides using the order he had just learned. It was hoped that the shape skipped would act as mediator for the following shape. Hence, this task will be referred to as mediation. The study slides were not shown again. Feedback was given as before, with the correct response for Slide 1 appearing in the center of Slide 2. The number of correct responses for a 
Table 1

Performance on Learning. Mediation, and Latency Tasks for Each Instruction Group

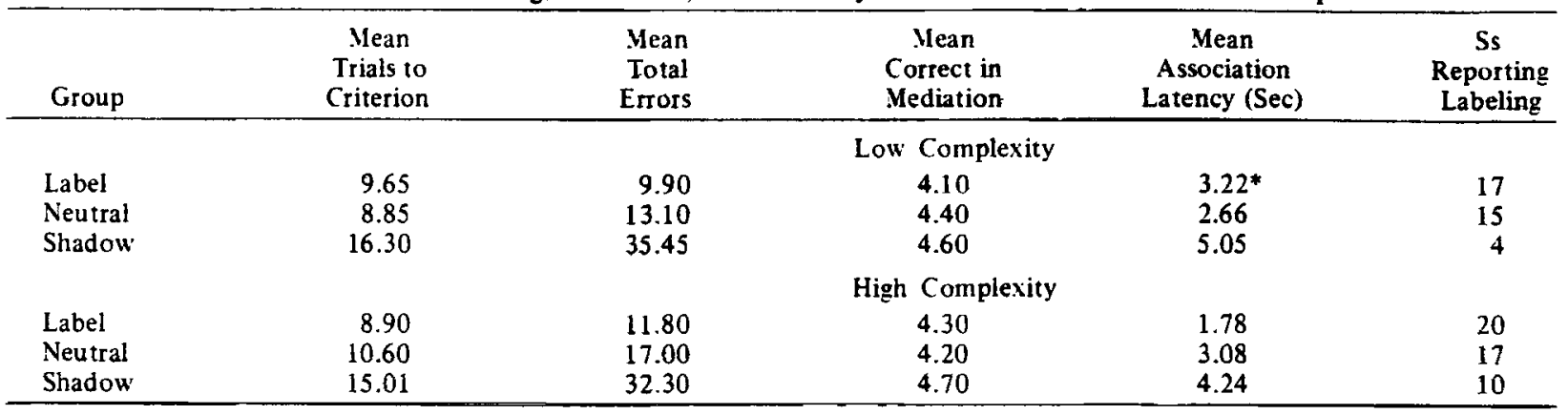

*1.35, eliminating $R N L s$

single test series was recorded, giving a total of five possible correct responses. The mediation task was identical for all three instruction groups. Group Sh did not shadow during this task.

\section{Association}

Following the mediation task, $\mathrm{S}$ was shown each study slide alone and asked to name the first thing that came to mind immediately upon seeing the shape; $S$ was told that if he had associated anything with the shape while learning the order, he should give such associations here. The time in 100 ths of seconds from the projection of the slide to S's response was recorded by means of a Standard Electric timer. Finally, $S$ was asked whether or not the associations he had given were also used in the earlier learning task. If not, he was asked to describe, if possible, how he had learned the order.

\section{Subjects}

The 120 Indiana University students used as Ss were assigned randomly to one of six conditions (three instruction sets and two levels of shape complexity). In addition, one $S$ was eliminated because of $E$ error, three because of failure to follow instructions, and three (all in the 24-point Sh condition) for failure to learn the serial task within 45 min. One $S$ was run in each condition before running further $S s$ in any other condition. Ss were given a choice of course credit or pay for serving; no differences in performance were noted between paid and nonpaid Ss.

\section{RESULTS AND DISCUSSION}

The mean number of errors per $S$ for each group is shown in Table 1. The two shadowing groups made many more errors than any other group; it has been well documented (Mowbray, 1953: Lindsay. Cuddy, \& Tulving, 1965) that the requirement to attend to two tasks simultaneously has an adverse effect on performance. The variances of both shadowing groups were significantly greater than the smallest variance in the other four groups $\left[F_{\max }(6,19)=4.14, p<.01\right]$. Consequently, these two groups were analyzed separately and were found not to differ significantly $[\mathrm{t}(38)=.12 . \mathrm{p}>.25]$.

Analysis of varance of mean errors for the remaining four groups revealed that the tendency for fewer emors among low-complexity groups was not significant $[F(1,76)=3.32, p>.05]$. The instructions effect was not reliable $[F(1,76)=1.59, p>.10)$, nor was the Complexity by Instructions interaction $[\mathrm{F}(1,76)=1.81$, $p>.10]$. Analysis of the number of trials to reach criterion (see Table 1) revealed the same pattern; the two shadowing groups took approximately the same number of trials to reach criterion, and there were no significant differences among the remaining four groups.

Previous investigators (e.g., Ellis \& Muller, 1964) have found an interaction between complexity and type of pretraining (observation and labeling), such that recognition of high-complexity shapes was superior for label pretraining, but recognition of low-complexity shapes was superior for observation pretraining. This two-way interaction was not significant in the present task, although there is a slight trend in that direction.

The results of this experiment do not indicate that ease of learning a serial list is reliably related to the use of labels. However, the conclusion depends upon the assumption that Ss in Group L used a labeling strategy more often than did Ss in Group N. It is possible that the serial task resulted in widespread use of labels, even when S was not explicitly instructed to use them. If so, we certainly would expect no differences between Groups $\mathrm{L}$ and $\mathrm{N}$, for they would be functionally equivalent. Further analysis revealed that this was the case, necessitating a shape-wise rather than S-wise analysis. The data were partitioned in two different ways in order to judge whether labeling a shape made it easier to learn. The first breakdown uses self-reports to categorize shapes as labeled or not.

\section{Verbal Reports}

For the most part, it seems reasonable to assume that Ss will know whether or not they have associated a name or description with a shape. Table 1 presents the number of $\mathrm{Ss}$ in each group who reported partial use of labels. There are two aspects of these verbal reports which can be used to argue for then validity. Consistent with Clark (1965). Ss reported labeling low-complexity shapes less 
Table 2

Mean Association Latency (in Seconds) and Mean Errors for Shapes Reported Labeled and Not Reported Labeled as a Function of Instructions and Complexity

\begin{tabular}{|c|c|c|c|c|c|c|}
\hline $\begin{array}{c}\text { Instruc- } \\
\text { tions }\end{array}$ & $\begin{array}{c}\text { Shapes } \\
\text { Reported } \\
\text { Labeled }\end{array}$ & $\begin{array}{c}\text { Mean } \\
\text { Association } \\
\text { Latency (Sec) }\end{array}$ & $\begin{array}{c}\text { Mean } \\
\text { Errors }\end{array}$ & $\begin{array}{c}\text { Shapes Not } \\
\text { Reported } \\
\text { Labeled }\end{array}$ & $\begin{array}{c}\text { Mean } \\
\text { Association } \\
\text { Latency (Sec) } \\
\end{array}$ & $\begin{array}{c}\text { Mean } \\
\text { Errors }\end{array}$ \\
\hline & \multicolumn{6}{|c|}{ Low Complexity } \\
\hline \multirow[t]{2}{*}{$\begin{array}{l}\text { Label } \\
\text { Neutral } \\
\text { Shadow* }\end{array}$} & $\begin{array}{l}82 \\
74 \\
17\end{array}$ & $\begin{array}{l}1.94 \\
2.10 \\
1.97\end{array}$ & $\begin{array}{r}2.26 \\
2.30 \\
10.00\end{array}$ & $\begin{array}{l}18 \\
26 \\
78\end{array}$ & $\begin{array}{l}9.46 \\
4.24 \\
5.86\end{array}$ & $\begin{array}{l}2.11 \\
2.54 \\
8.58\end{array}$ \\
\hline & \multicolumn{6}{|c|}{ High Complexity } \\
\hline $\begin{array}{l}\text { Label } \\
\text { Neutral } \\
\text { Shadow* }\end{array}$ & $\begin{array}{r}100 \\
83 \\
47 \\
\end{array}$ & $\begin{array}{l}1.74 \\
2.84 \\
2.12\end{array}$ & $\begin{array}{l}2.62 \\
3.73 \\
7.55\end{array}$ & $\begin{array}{r}0 \\
17 \\
48 \\
\end{array}$ & $\begin{array}{c}- \\
4.27 \\
6.04\end{array}$ & $\begin{array}{l}- \\
1.76 \\
6.17 \\
\end{array}$ \\
\hline
\end{tabular}

*Records of one $S$ 's verbal report lost.

often than high-complexity shapes. Secondly, labeling was reported most often in those groups instructed to label and least often in shadowing groups, where it was expected that labeling would be discouraged by the interference introduced into the verbal channel. Hence, the pattern of verbal reports corresponds to previous reports and to expectations based upon experimental manipulations.

It might also be noted that, when questioned, most Ss had little doubt about what $\mathrm{E}$ was asking, and they displayed little indecision about their answers. Many Ss who denied using labels had ready explanations of their strategies in terms of size, directionality, or dynamic relations among the shapes (e.g., rotating or fitting the shapes together).

Furthermore, the association task provided a converging operation for assessing S's use of labels. Given the instructions, Ss should have responded with any label or association used in the learning task, and a short latency should generally indicate that a particular shape had been labeled; conversely, if a shape had not been labeled, then a longer latency would reflect S's search for an association. Shapes which $S$ claimed to have labeled had much shorter latencies (see Table 2) on the association task than those shapes which Ss denied labeling.

Of course, one cannot tell at what point during learning a shape was labeled, but presumably most shapes are labeled in advance of attainment of the serial order. If labels are an aid in learning to recognize a shape, then those shapes labeled should have had fewer errors than those which were not. Table 2 reports the number of shapes reported as labeled and nonlabeled for each group, along with the number of errors committed during serial learning. Although reporting data in such a fashion confounds $\mathbf{S}$ differences with item differences, and although means are based on unequal ns, for four of five comparisons, fewer errors were committed on the shapes which Ss claimed not to have labeled during learning. A conclusion consistent with these results is that recognition of a shape is not facilitated because $S$ has a verbal association to it. Eleven Ss reported only partial labeling. For these 11 , we determined the mean error rate for the labeled shapes and the nonlabeled shapes. The means were exactly the same for $3 \mathrm{Ss}$; the error rate was smaller for labeled shapes in four of the remaining eight cases. The mean number of errors on labeled shapes (per S) was 5.09 , vs 4.74 for nonlabeled shapes.

\section{Association Latency}

A second breakdown depends solely upon association latency; the mean for each instruction group is reported in Table 1 . The high latency for the low-complexity $L$ group was caused by the presence of four Ss who stated that they did not use labels, contrary to instructions. The mean latency excluding these four Ss was 1.35, more in line with expectations. Analysis of variance revealed instructions to be the only significant factor $[F(2,114)=12.41, p<.01]$. A Newman-Keuls analysis indicated that the high-complexity $\mathrm{Sh}$ group differed significantly from the high-complexity L group $(p<.05)$. Only the low-complexity Sh group differed reliably from both $\mathrm{L}$ groups and both $\mathrm{N}$ groups $(p<.05)$. The two Sh groups did not differ from each other.

If a long latency is assumed to be indicative of a nonlabeler, then the preponderance of long latencies in Group Sh attests to the effectiveness of the shadowing task as an inhibitor of labeling. The rank ordering of latencies corresponds to the rank ordering of number of Ss reporting no labeling (RNLs) present in Groups L (3), $N(8)$, and Sh (26), collapsing over complexity.

The lack of a significant latency difference between the high-complexity Sh group and the low-complexity L and both $\mathrm{N}$ groups can perhaps be explained by noting the number of reported labelers (RLs) and RNLs in each group. All Ss in the high-complexity $L$ group reported using labels; however, some $S s$ in all other groups reported using no labels. The greatest number of RNLs was in the low-complexity Sh group (16 of 20). The high-complexity Sh group had 10 RNLs, while the number of RNLs for the high-complexity $\mathrm{N}$ group, the 
low-complexity $\mathrm{N}$ group. and the low-complexity $\mathrm{L}$ group were 3.5 . and 3 . respectively. The high-complexity $\mathrm{Sh}$ group. lying between the two extreme numbers of RNLs present. did not differ from the extremes, but only from the group with no RNLs. The low-complexity Sh group, having almost all RNLs, does differ from all groups except the high-complexity Sh groups. This explanation, based on Ss' reports of using or not using labels, assumes that the RL latencies were shorter than RNL latencies, an apparently valid assumption.

Since all RLs did not consistently label every shape and since all RNLs did not consistently learn without labeling. a comparison of errors for short- and long-latency items in original learning was made. Long-latency items were defined as those having latencies greater than $4.00 \mathrm{sec}$; otherwise, the item was considered to have a short latency. This figure was chosen as the cutoff value between short and long latencies because, in the comparison of RL and RNL mean latencies, rarely was an RL mean latency above 4.00 and rarely was an RNL mean latency below 4.00 . There were 460 items with latencies less than 4.00 and 140 items with latencies greater than 4.00 . The percentage of long-latency items $(23 \%)$ was less than the percentage of RNL Ss (30\%). However, taking into account the means and distributions of mean latencies of RLs and RNLs, 4.00 seemed to be the best cutoff point.

Table 3 presents the number of long- and short-latency items for each group. along with the mean number of errors made on that set of items during serial learning. The differences among error rates are small and not consistent. There is no general tendency for short-latency items to be learned with fewer errors than items with long latencies.

Whether or not a single item was encoded in terms of a label (as indicated by latency of association) appeared to be unrelated to the number of errors made when learning that item. All types of encoding devices used by Ss, whatever they were, proved equally efficient in the learning of visual nonsense shapes.

\section{Mediation}

The mean total correct in the mediation task for each instruction group is reported in Table 1 . The mediation scores for all groups were quite high (total possible correct was 5). Analysis of variance revealed no significant differences due to any of the variables. Although Sh groups learned more slowly and with more errors than did the $\mathrm{L}$ or $\mathbf{N}$ groups, the shape order was retained just as well.

\section{CONCLUSION}

None of the analyses reported can be construed as indicating that labeling a shape facilitates learning position in a serial list. This is not a surprising result. It
Table 3

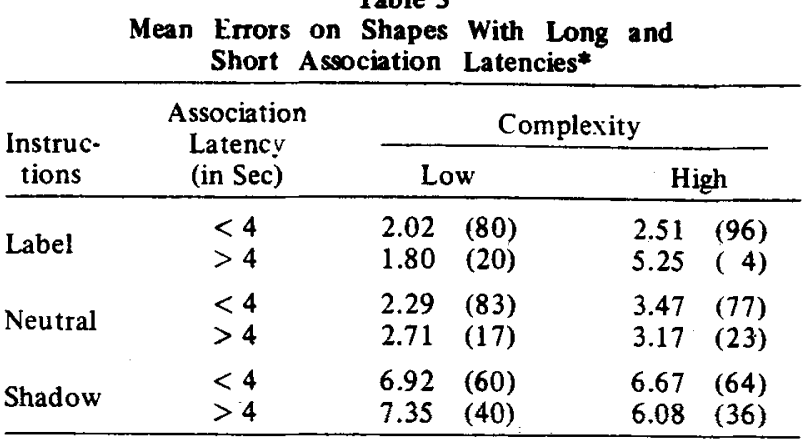

* Number of shapes in parentheses

has been suggested (cf. Daniel \& Ellis, 1972; Hake \& Eriksen, 1955) that the advantage of label pretraining with relevant labels (in stimulus predifferentiation studies) is to direct S's attention towards those aspects most helpful in discriminating that shape from the others. Hence, task requirements favor labeling pretraining over observation pretraining because of the amount of stimulus discrimination required. However, the pretraining paradigm does not insure equal amounts of attention for labeling and observation Ss; the serial-list procedure does. The serial-list procedure requires that all shapes be intensively searched for the aspects which will support correct performance. Whether or not a label is attached is superfluous: labels arise as a result of the effort to discriminate the stimuli, and once a stimulus is discriminated, having a label makes no difference.

These remarks must be tempered somewhat, since it appears that labels can make a difference even when pretraining equates amount of attention (Malloy \& Ellis, 1970; Santa \& Ranken, 1972). However, these studies also used somewhat atypical testing procedures. It is not altogether clear why labels were irrelevant in the serial task, but not in those tasks used by Malloy and Ellis (1970) and Santa and Ranken (1972).

\section{REFERENCES}

Clark, H. J. Recognition memory for random shapes as a function of complexity, association value and delay. Journal of Experimental Psychology, 1965, 69, 590-595.

Daniel, T. C., \& Ellis, H. C. Stimulus codability and long-term recognition memory for visual form. Journal of Experimental Psychology, 1972. 93. 83-89.

Ellis, H. C. Transfer of stimulus predifferentiation to shape recognition and identification learning: Role of properties of verbal labels. Journal of Experimental Psychology. 1968, 78. $401-409$.

Ellis. H. C. Transfer and retention. In M. H. Marx (Ed.). Learning: Processes. New York: Macmillan. 1969.

Ellis. H. C.. \& Homan. L. E. Implicit verbal responses and the transfer of stimulus predifferentiation. Journal of Experimental Psychology. 1968. 76. 486-489.

Ellis. H. C.. \& Muller. D. G. Transfer in perceptual learning following stimulus predifferentiation. Journal of Experimental Psychology. 1964.68. 388-395 
Epstein. W. Varieties of perceptual learning. New York: MoGraw-Hill, 1967.

Hake, H. W., \& Eriksen, C. W. Role of response variables in recognition and identification of complex visual forms. Journal of Experimental Psychology, 1956, 52. 235-243.

Lindsay, P. H., Cuddy, L. L., \& Tulving. E. Absolute judgments of simultaneously presented visual and auditory stimuli. Psychonomic Science, 1965, 2, 211-212.

Malloy, T. E., \& Ellis, H. C. Attention and cue-producing responses in response-mediated stimulus generalization. Journal of Experimental Psychology, 1970, 83, 191-200.

Martin, E. Stimulus meaningfulness and paired-associate transfer: An encoding variability hypothesis. Psychological Review. $1968,75,421-441$.

Mowbray, G. H. Simultaneous vision and audition: The comprehension of prose passages with varying levels of difficulty. Joumal of Experimental Psychology, 1953, 46, 365-372.

Ranken, H. B. Language and thinking: Positive and negative effects of naming. Science, 1963, 141, 48-50.
Robinson, J. S. The effect of learning verbal labels for stimuli on their later discrimination. Journal of Experimental Psychology, 1955. 49. 112-114

Rock, I., Lasker, A.. \& Simon, J. Stimulus generalization as a process of recognition. American Journal of Psychology, $1969,82,1-22$.

Santa, J., \& Ranken. H. Effects of verbal coding on recognition memory. Journal of Experimental Psychology, 1972, 93, 268-278.

Vanderplas, J. M., \& Garvin, E. A. The association value of random shapes. Journal of Experimental Psychology, 1959, $57,147-154$

Vanderplas, J. M., Sanderson. W. A., \& Vanderplas, J. N. Some task-related determinants of transfer in perceptual learning. Perceptual \& Motor Skills. 1964, 18, 71-80.

(Received for publication June 9, 1972; second revision accepted November 22, 1972.) 